\title{
Role of Antibiotics in Neonates Born through Meconium Stained Amniotic Fluid
}

\author{
Ravi Tiwari*, Om Shankar Chaurasiya**, Sapna Gupta***, \\ Sanjaya Sharma****, Mayank Singh***** \\ *Junior Resident, Department of Paediatrics, M L B Medical College Jhansi \\ ** Associate Professor and Head, Department of Paediatrics, M L B Medical College Jhansi \\ ***Associate Professor, Department of Paediatrics, M L B Medical College Jhansi \\ *****Professor and Head, Department of Obstetrics and Gynecology, M L B Medical College Jhansi \\ ******Professor and Head, Department of Pathology, M L B Medical College Jhansi
}

Corresponding Author: Om Shankar Chaurasiya

\begin{abstract}
The objective of our study was to compare the clinical course and infection related outcome in cases of meconium stained amniotic fluid neonates treated with or without antibiotic therapy. One hundred fifty eligible neonates were randomized to antibiotic group and non antibiotic group. Both groups were compared for development of sepsis screen and final outcome in both groups were compared.

The incidence of sepsis screen positive was observed in both groups. In antibiotic group sepsis screen was positive in 5 cases and in non antibiotic group sepsis screen was positive in 4 cases. Overall incidence of sepsis screen positive was similar in both antibiotic and non antibiotic group.

The difference was not found to be statistically significant $(\mathrm{p}$ value $=1.00$ and chi square value 0.118 ).

Final outcome in both antibiotic group and non antibiotic group showed that in antibiotic group, out of 75 neonates 71 were discharged successfully and 4 were died. In non antibiotic group, 73 were discharged successfully and 2 died, out of 75 neonates. There was statistically no significant difference on final outcome between these two groups ( $\mathrm{p}$ value 0.68 and chi square value 0.694 ).

Conclusion: There was no difference in the incidence of infection and in final outcome in neonates born through meconium stained amniotic fluid treated with or without antibiotics.
\end{abstract}

Keywords: Meconium Stained Amniotic Fluid, Neonates, Antibiotic Therapy

\section{INTRODUCTION}

Meconium passage in newborn infants is a developmentally programmed event normally occurring within first 24-48 hrs of birth. The meconium stained amniotic fluid as a result of passage of fetal caloric contents into the amniotic cavity is noted in approximately $13 \%$ of all deliveries ${ }^{(1,2)}$.

Meconium aspiration syndrome develops in $5 \%$ of infants delivered through meconium stained amniotic fluid. More than $4 \%$ of meconium aspiration syndrome infants die accounting for $2 \%$ of all perinatal deaths ${ }^{(1,3)}$.

The routine use of antibiotics in meconium stained amniotic fluid babies has been advocated for a long time as a part of conventional treatment. Meconium passage in utero is hypothesized to represent a response to fetal bacterial infection ${ }^{(4)}$. Experimental works have shown that meconium enhances bacterial growth in vitro $^{(5,6)}$ and the risk of intraamniotic infection is increased in the presence of meconium stained amniotic fluid ${ }^{(7)}$.

The incidence of bacterial infection in neonates born through meconium stained amniotic fluid as well as those developing meconium aspiration syndrome has not been systematically evaluated till date ${ }^{8)}$. 
With the rising concern about the emergence of resistant strains in neonatal intensive care units and the possible side effects of antibiotics (like aminoglycosides) including nephrotoxicity and ototoxicity in neonates a systematically conducted, randomized controlled trial is necessary to assess the utility of antibiotics in the routine management of infants with meconium stained amniotic fluid.

\section{MATERIAL AND METHODS}

This study was prospective open labelled study that included 150 neonates admitted in SNCU of Department of Pediatrics, MLB Medical College, Jhansi.

Period of study - May 2018 - August 2019

Data collection and Sample size - 167 neonates admitted in SNCU born through MSAF, after inclusion and exclusion criteria 150 were eligible for study.

\section{Method:}

Inclusion criteria: All newborn babies born through meconium stained amniotic fluid admitted within $48 \mathrm{hrs}$ of birth.

Exclusion criteria: Presence of major congenital malformation, refusal of consent by the parents and newborn with either clinical history suggestive of sepsis in mother or investigation suggestive of sepsis in newborn at the time of admission were excluded.

Parents of the eligible neonates were approached for participation and subjects were enrolled only after obtaining a written informed consent in their own local language.

Enrolled neonates were randomized into the antibiotic group and non antibiotic group based on randomization, done using Lottery system.

Antibiotic Group - 75 neonates randomized to receive prophylactic intravenous antibiotics according to firstline antibiotic policy of the unit.

Non antibiotic group - 75 neonates not received any antibiotics.

Since the mid 1970s, interventions to decrease the mortality and morbidity of meconium aspiration syndrome in infants who are born through meconium stained amniotic fluid have been recommended. The practice of universal oropharyngeal suctioning of the fetus on the perineum followed by routine intubations and suctioning of the trachea at birth was generally practices for many years. This practice was abandoned over a decade ago after a large multicentre, multinational randomized clinical trial provided evidence that newborns born through meconium stained amniotic fluid who were vigorous as birth did not benefit from intervention and could avoid the risk of intubation.

Because the presence of meconium stained amniotic fluid may indicate fetal distress and increases the risk that the infant will require resuscitation after birth a team that includes an individual skilled in tracheal intubation should be present at the time of birth. If the infant is vigorous with good respiratory efforts and muscle tone, the infant may stay with the mother to receive the initial steps of newborn care. Gently clearing of meconium from the mouth and nose with a bulb syringe may be done in necessary.

However, if the infant born through meconium stained amniotic fluid presents with poor muscle tone and inadequate breathing efforts, the initial step of resuscitation should be completed under the radiant warmer. PPV should be initiated if the infant is not breathing or the heart rate is less than 100/min after the initial steps are completed. Routine intubation for tracheal suction in this setting is not suggested, because there is insufficient evidence to continue recommending this practice.

A detailed antenatal history was elicited to find out the etiology of passage of meconium into amniotic fluid. Detailed natal history and presenting complaints were also taken. In all meconium stained infants APGAR score at $1 \mathrm{~min}$ and $5 \mathrm{~min}$ were assessed and birth weight, gestational age (by Ballard scoring) and respiratory distress (by Downey's score) was seen.

Detailed examination of the newborn was done with regard to gestational age 
estimation, anthropometric measurement, general examination and systemic examination.

Estimation of gestational age was done by recording date of LMP and confirmed by New Ballard scoring which includes neuromuscular maturity and physical maturity. Anthropometric measurement includes weight, length, head and chest circumference was recorded in each case.

General examination along with vitals, detailed examination was carried out in each case to evaluate the general appearance (posture, activity, cry, colour) head (caput, cephalohematoma, fontanels, sutures, nose for nasal flaring and choanal atresia and mouth for cleft lip and palate) neck (masses and swelling) chest (shape, respiratory effort, apex beat which position shifted in case of cardiomegaly, Abdomen, genitals and limbs.

Primitive reflexes (Moro's rooting, sucking and grasp reflex) were also evaluated in each case.

Meconium staining can be appreciated on finger nails, umbilical cord and vernix caseosa (skin). Meconium staining in utero may be a due to an infant who was stressed before birth.

Meconium stained finger nails- normally the nails are white in colour, yellow tints on nail suggest meconium staining which indicates meconium was present in utero some time prior to delivery.

Meconium stained umbilical cord normally umbilical cord is light yellow colour but presence of meconium in amniotic fluid gives a dark green colour.

Meconium stained vernix caseosa (skin) it is not the skin itself but the vernix that shows the evidence of meconium in amniotic fluid. Vernix caseosa is the pasty, cheese like creamy white colour material present in varying degrees on skin at birth. Yellow brown tint in vernix indicates meconium staining in utero.

Systemic examination - A detailed systemic examination was undertaken in each and every case with special emphasis on respiratory system, under the following headings.

Inspection - Inspection of thorax was done both anteriorly and posteriorly, first movement of chest wall (symmetry, presence of retractions) and the use of abdominal or accessory muscles in respiration. Next focus was the shape of chest wall and obvious deformities. Finally vertebral column was inspected for any irregularities. Further inspection of nose for nasal flaring and mucous membrane of mouth and lips for the presence of central cyanosis was carried out. Nails beds were examined for evidence of peripheral cyanosis.

Palpation - Trachea was palpated in suprasternal notch for morbidity and deviation from midline.

Percussion - Usually not very informative. Auscultation - chest wall was auscultated by using diaphragm of paediatric stethoscope air entry (symmetry and adequacy of air exchange) characterization of the normal breath sounds (as bronchial bronchovesicular or vesicular) and adventitious sounds (such as crepitations, wheeze or rubs) was done.

After examination of respiratory system a detailed cardiovascular and central nervous system examination was done in each and every case.

All babies irrespective of their group allocation were admitted to the nursery and worked up for sepsis using a sepsis screen and blood culture. Sepsis screen consisting of total leukocyte count, absolute neutrophil count and immature to total neutrophil ratio (by Coulter and peripheral smear examination), micro-ESR and C-reactive protein will be performed at admission or thereafter if required. Blood culture was performed at admission and thereafter if required. Symptomatic babies (presence of respiratory distress, lethargy, abdominal distension, temperature or hemodynamic instability, hypoglycemia, apnea, or any other systemic abnormalities), either from birth or any time during the course of stay, in both groups, were subjected to further 
investigations such as chest X-ray, arterial blood gas, and lumbar puncture as deemed necessary by the treating physician. Appropriate treatment was started or modified as per the decision of consultantin- charge taken as one serving the best interest of the baby. All such cases requiring prolongation of antibiotics beyond 3 days in Antibiotic group, or starting of antibiotics in the no Antibiotic group (symptomatic or sepsis screen positive), were noted. All babies received supportive care in the form of maintenance of temperature, fluid balance, and blood glucose. Further respiratory, cardiac, or other system support as needed was provided as per standard unit protocol. All these neonates were monitored daily by the study coordinators for vital signs, i.e., heart rate, respiratory rate, blood pressure, oxygen saturation, and signs of respiratory distress or failure till the time of discharge (minimum 72 hours) or death. Neonates who were discharged were advised for followed up.

Data pertaining to various maternal demographic variables like parity, risk factors for sepsis (prolonged rupture of membranes $>24 \mathrm{~h}$, intrapartum fever $\geq 38.0$ ${ }^{\circ} \mathrm{C}$, unclean or frequent per-vaginal examination $(\geq 3)$, clinical chorioamnionitis, maternal UTI), fetal distress (fetal heart rate abnormalities on auscultation or cardiotocography), meconium consistency (thick pea soup or thin watery), mode of delivery, along with neonatal variables like sex, birth weight, gestational age, APGAR score, incidence of non-vigorous neonates and requirement of endotracheal intubation for positive pressure ventilation was recorded in a pretested proforma. Additional data collected during neonatal hospital stay included, duration and severity of respiratory distress (using Downe's score), requirement and total duration of oxygen therapy, need for and duration of CPAP or mechanical ventilation, and incidence of complications like air leaks or persistent pulmonary hypertension of newborn (PPHN). In addition, any development or progression of hypoxic ischemic encephalopathy (HIE) or involvement of other organ systems and the duration of stay was also recorded.

\section{RESULT}

During period of study that is from May 2018 to August 2019, 167 babies born through meconium stained amniotic fluid were admitted to SNCU out of them 17 were excluded, out of which 6 due to refusal of consent, 2 due to presence of major congenital malformation and 9 due to clinical history suggestive of sepsis as per exclusion criteria. Only 150 babies are eligible for study.

So this study include 150 neonates born through meconium stained amniotic fluid out of 150 neonates, 75 neonates in antibiotic group received antibiotics and 75 neonates in non antibiotic group not receive any antibiotic.

Selected baseline variable are represented in table given below. There was no difference between the groups with regard to gestational age, gender, birth weight consistency of meconium. Time of onset of respiratory distress, respiratory distress scoring, APGAR score, sepsis screen, vigorous and non vigorous state and final outcome.

\section{Baseline variables}

\begin{tabular}{|l|l|l|}
\hline Parameters & Antibiotic group & Non antibiotic group \\
\hline Sex & & \\
\hline Male & 53 & 41 \\
\hline Female & 22 & 34 \\
\hline Gestational age & & \\
\hline$<35$ wks & 9 & 7 \\
\hline $36-38$ wks & 43 & 38 \\
\hline $39-40$ wks & 23 & 29 \\
\hline $41-42$ wks & 0 & 1 \\
\hline Birth weight & & \\
\hline$<2.5 \mathrm{~kg}$ & 12 & 16 \\
\hline $2.5-3.5 \mathrm{~kg}$ & 62 & 57 \\
\hline$>3.5 \mathrm{~kg}$ & 1 & 2 \\
\hline
\end{tabular}




\begin{tabular}{|c|c|c|}
\hline \multicolumn{3}{|c|}{ Table Continued... } \\
\hline Arterial blood gas analysis & & \\
\hline Arterial pH (mean \pm SD) & $7.16 \pm 0.18$ & $7.19 \pm 0.16$ \\
\hline Base excess (mean \pm SD) & $-12.13 \pm 6.09$ & $-10.88 \pm 6.63$ \\
\hline \multicolumn{3}{|l|}{ Consistency of meconium } \\
\hline Thick & 17 & 14 \\
\hline Thin & 58 & 61 \\
\hline Vigorous & 58 & 63 \\
\hline Non vigrous & 17 & 12 \\
\hline \multicolumn{3}{|l|}{ Respiratory distress scoring } \\
\hline$<4$ & 67 & 69 \\
\hline $4-6$ & 6 & 5 \\
\hline$>6$ & 2 & 1 \\
\hline \multicolumn{3}{|c|}{ Time of onset of respiratory distress } \\
\hline Absent & 1 & 41 \\
\hline Onset $<2 \mathrm{hrs}$ & 60 & 56 \\
\hline Onset 2-4 hrs & 12 & 14 \\
\hline Onset $>6 h r s$ & 2 & 4 \\
\hline \multicolumn{3}{|l|}{ Apgar score at $1 \mathrm{~min}$} \\
\hline $0-3$ & 5 & 3 \\
\hline $4-7$ & 56 & 54 \\
\hline$>7$ & 14 & 18 \\
\hline \multicolumn{3}{|l|}{ Sepsis screen } \\
\hline Positive & 5 & 4 \\
\hline Negative & 70 & 71 \\
\hline \multicolumn{3}{|l|}{ Final outcome } \\
\hline Discharge & 71 & 73 \\
\hline Expire & 4 & 2 \\
\hline
\end{tabular}

\section{Primary outcome}

All babies were assessed for development of sepsis in antibiotic group and non antibiotic group.

The incidence of sepsis screen positive was observed in both the groups.

In antibiotic group sepsis screen was positive in 5 cases and in non antibiotic group sepsis screen was positive in 4 cases.

Overall incidence of sepsis screen positive was similar in both antibiotic and non antibiotic group. This difference was not found to be statistically significant. ( $p$ value $=1.00$ and chi square value 0.118 ) .

In antibiotic group all patients received antibiotics and in non antibiotic group, antibiotic were added in 17 patients. The reason for starting of antibiotic in 17 patients of non antibiotic group were positive sepsis screen in 4 patients, positive blood culture in 7 patients and clinical condition suggestive of infection in 6 patients.

\section{Secondary outcome}

In antibiotic group and in non antibiotic group no significant difference was detected in time of onset and severity of respiratory distress.
Time of onset of respiratory distress in both antibiotic group and non antibiotic group was found statistically not significant. ( $\mathrm{p}$ value 0.80 and chi square 0.958 ).

Severity of respiratory distress on basis of Downe's /Silverman scoring, found statistically no significant different between antibiotic group and non antibiotic group ( $p$ value 0.79 and chi square value 0.454 ).

During course of study total 6 patients died. In antibiotic group 4 patients died and in non antibiotic group 2 patients died.

Final outcome in both antibiotic group and non antibiotic group showed that in antibiotic group, out of 75 patients 71 were discharged successfully and 4 were died, and in non antibiotic group 73 were discharged successfully and 2 died. There was statistically no significant difference on final outcome between these two groups ( $p$ value 0.68 and chi square value $=0.694$ )

\section{DISCUSSION}

This study shows that routine administration of prophylactic antibiotics had no demonstrable benefit in the management of neonates born through meconium stained amniotic fluid, especially 
in asymptomatic neonates. The incidence of infections and respiratory complications is not altered by antibiotic use and the overall mortality or morbidity of such neonates is also not affected by this intervention. In both groups, incidence of sepsis and mortality are comparable.

Presence of meconium in amniotic fluid has long been speculated to increase the susceptibility of neonates to sepsis. Studies in the past have shown the likelihood of fetal bacterial infection as a causative factor for passage of meconium. Moreover in vitro studies done by Florman et $\mathrm{al}^{6}$ (1969), Bryan et $\mathrm{al}^{5}$ (1967) have demonstrated that meconium alters the bacteriostatic properties of amniotic fluid predisposing the fetus to infection. This increased susceptibility to infection has been believed to result from decreased host immune response as demonstrated by Craig et $\mathrm{al}^{9}$. The difficulty in differentiating bacterial pneumonia from meconium aspiration syndrome on the basis of clinical and radiographic findings prompts physicians globally to have a low threshold for starting antibiotics in infants with meconium stained amniotic fluid.

Recent studies have however challenged this concept both in terms of overall incidence of sepsis and the role of antibiotic in reducing or preventing infectious morbidity in neonates born through meconium stained amniotic fluid Wiswell et $\mathrm{al}^{8}$ (2004) examined the incidence of culture proven bacterimia in 741 meconium stained neonates and demonstrated the incidence of culture proven bacteremia to be $0.7 \%$ in meconium stained amniotic fluid population compared with $0.8 \%$ in babies born through clear liquor.

In a retrospective analysis by Singh et $\mathrm{al}^{13}$ (1995), positive blood culture was obtained only in $2.5 \%$ of neonates born with meconium aspiration syndrome. Similarly Krishnan et $\mathrm{al}^{14}$ (1995) in their retrospective review, found no significant difference in the incidence of septicemia, between infants intubated for intratracheal suctioning of meconium compared to non intubated infants. These three studies reveal that incidence of sepsis is not significantly high in meconium stained neonates.

Studies evaluating the role of antibiotic found no difference in infections morbidity in babies with meconium aspiration morbidity in babies with meconium aspiration syndrome, treated with or without antibiotics, in the studies by Lin et $\mathrm{al}^{10}$ (2005) and Shankar et $\mathrm{al}^{12}$ (1995). Similarly the incidence of sepsis in MAS reported by Basu et $\mathrm{al}^{10}$ (2007) was $4 \%$ in the antibiotic and $2.7 \%$ in no antibiotic group.

In the present study, incidence of sepsis screen positive was $7 \%$ and $5 \%$ respectively in antibiotic group and non antibiotic group. Further no difference in severity of respiratory distress and mortality was noted between the two groups. Similarly to the results obtained in other study mentioned above.

The study by Shankar et $\mathrm{al}^{12}$ had a very small sample size, excluded neonates with maternal risk factors for neonate sepsis and did not define sepsis based on blood culture positivity. Basu et $\mathrm{al}^{9}$ in their study of infants with meconium aspiration syndrome excluded all infants with any risk factor for sepsis and those who developed early onset sepsis within firs $24 \mathrm{hrs}$ of life. Moreover, they randomized the subjects at $24 \mathrm{hr}$ of life thus compromising the generalizability of the study. No follow up was done in both of these studies.

A more extensive study by Lin et $\mathrm{al}^{11}$ though comparable in sample size to our study included only non ventilated cases of meconium aspiration syndrome with no perinatal risk factor for sepsis. All these studies have included neonates without risk factors for sepsis and have reported no benefit of antibiotic administration for management of meconium aspiration syndrome. Out study has comprehensively included all meconium stained neonates irrespective of risk status for sepsis and yet the results reveal no role of antibiotic in their management. 


\section{CONCLUSION}

The zest of our study was that there was no statistical significant difference in the incidence of infection in neonates born through meconium stained amniotic fluid treated with or without antibiotics and no difference in final outcome in neonates born through meconium stained amniotic fluid treated with or without antibiotics.

The present study also supports and condemns the unnecessary use of antibiotics without evidence of sepsis, so as to prevent antibiotic resistance that is rising to dangerously high levels in all parts of the world. Further this prophylactic use of antibiotics may leads to longer hospital stay, high medical cost and increased mortality.

Therefore we suggest the avoidance of empirical use of antibiotics in such neonates born through meconium stained amniotic fluid without documented evidence of infections.

\section{Acknowledgement: None}

\section{Conflict of Interest: None}

\section{Source of Funding: None}

\section{Ethical Approval: Approved}

\section{REFERENCES}

1. Cleary GM, Wiswell TE. Meconium stained amniotic fluid and the meconium aspiration syndrome an update. Pediatr Clin North AM, 1992; 45 : 511-529.

2. Wiswell TE. Advances in the treatment of the meconium aspiration syndrome. Acta Paediatr, 2001; 90 (Suppl) : 28-30.

3. Wiswell TE, Tuggle JM, Turner BS. Meconium aspiration syndrome. Have we made a difference? Pediatrics, 1990; 85: 715-721.

4. Blot P, Milliez J, Breart G, Vige $P$, Nessmann C, Onufryk JP, Dendrinos S, Surran C. Fetal tachycardia and meconium staining - a signs of fetal infection . Int $\mathbf{J}$ Gynecol Obstet, 1983; 21: 189-194.
5. Bryan CS. Enhancement of bacterial infections by meconium. Johns Hopkins Med J, 1967; 121: 9-13.

6. Florman AL, Teubner D. Enhancement of bacterial growth in amniotic fluid by meconium. J Pediat. 1969; 74 : 111-114.

7. Romero $\mathrm{R}$, Hanaoka $\mathrm{S}$, Mazor $\mathrm{M}$, Athanassiadis AP, Callahan R, Hsu YC, Avila C, Nores J, Jimenez C. Meconium stained amniotic fluid : A risk factor for microbial invasion of the amniotic cavity. Am J Obstet Gynaeol. 1991; 163 : 859-862.

8. Wiswell TE, Henley MA. Intratracheal suctioning, systemic infection and the meconium aspiration syndrome. Pediatrics. 1992; 89: 203-207.

9. Craig S, Lopez A, Hoskin D, Markham F. Meconium inhibits phagocytosis and stimulates respiratory burst in alveolar macrophages. Pediatr Res. 2005; 67(6) : 813-818.

10. Basu S, Kumar A, Bhati BD. Role of antibiotics in meconium aspiration syndrome. Ann Trop Pedaitr. 2007; 27 : 107-113.

11. Lin HC, Sy BH, Tsai CH, Lin TW, Yeh TF. Role of antibiotics in management of non ventilated cases of meconium aspiration syndrome without risk factors for infection. Biol Neonate. 2005; 87 : 51-58.

12. Shankar V, Paul VK, Deorari AK, Singh M. Do Neonates with meconium aspiration syndrome require antibiotics? Indian $\mathrm{J}$ Pediatr. 1995; 62: 327-331.

13. Singh BS, Clark RH, Powers RJ, Spitzer AR. Meconium aspiration remains a significant problem in the NICU : Outcomes and treatment patterns in term neonates admitted for intensive care during a ten year period. J. Perinatal. 2009; 29: 497-503.

14. Krishnan L, Prabhakar P, Bhaskaranand N : Routine antibiotic cover for newborns intubated for aspirating meconium: is it necessary? Indian Pediatr. 1995; 32(5): 526531.

How to cite this article: Tiwari R, Chaurasiya OS, Gupta $S$ et.al. Role of antibiotics in neonates born through meconium stained amniotic fluid. International Journal of Research and Review. 2021; 8(9): 142-148. DOI: https://doi.org/10.52403/ijrr.20210920 